\title{
Left ventricular dyssynchrony in diabetes mellitus
}

\author{
Yogita Rochlani, MD, a Mohammed Hasan Khan, MD, ${ }^{\text {a }}$ Perry Gerard, $M D,{ }^{b}$ and \\ Diwakar Jain, $M^{c}$ \\ a Division of Cardiology, New York Medical College/Westchester Medical Center, Valhalla, NY \\ b Division of Nuclear Medicine, New York Medical College/Westchester Medical Center, \\ Valhalla, NY \\ c Division of Cardiology and Nuclear Medicine, New York Medical College/Westchester Medical \\ Center, Valhalla, NY
}

Received Oct 26, 2018; accepted Oct 26, 2018

doi: $10.1007 / \mathrm{s} 12350-018-01519-5$

\section{See related article, pp. pp. 1640-1648}

Myocardial perfusion imaging (MPI) with gated single-photon emission computed tomography (SPECT) or positron emission tomography (PET) is used for the detection of myocardial perfusion abnormalities and for the evaluation of left ventricular function. Automated programs exist for the estimation of ejection fraction as well as measuring scar size and ischemia. These programs are objective, highly reproducible, and reliable. ${ }^{1-5}$ Gated SPECT MPI is used for the diagnosis and risk stratification of patients with known or suspected coronary artery disease. In 2005, Chen et al. described the technique of phase analysis with gated SPECT imaging to assess the synchrony of left ventricular contraction. ${ }^{6}$ The assessment of left ventricular synchrony using phase analysis with gated SPECT adds another dimension to cardiac assessment using gated SPECT. This technique has evolved over the last few years making SPECT MPI a powerful tool that allows for the evaluation of perfusion, ventricular function and synchrony of mechanical contraction.

Left ventricular dyssynchrony is seen in the presence of conduction abnormalities such as left bundle branch block, right bundle branch block, ventricular

Reprint requests: Yogita Rochlani, MD, Division of Cardiology, New York Medical College/Westchester Medical Center, 100 Woods Road, Macy Pavilion, Valhalla, NY 10595; yogita.rochlani@gmail.com

J Nucl Cardiol 2020;27:1649-51.

$1071-3581 / \$ 34.00$

Copyright (c) 2018 American Society of Nuclear Cardiology. pacing, left ventricular dysfunction, and heart failure. Left ventricular dyssynchrony has been studied using several imaging modalities including echocardiography (M-mode, color tissue Doppler, strain imaging, and 3D echo), myocardial perfusion imaging (SPECT and PET), gated blood pool imaging, and cardiac magnetic resonance imaging. ${ }^{7}$ Dyssynchrony analysis using gated SPECT imaging has several advantages over the other modalities including use of automated technology, ease of standardization to ensure reliability and reproducibility, and lack of limitations such as requiring adequate windows as in the case of echocardiography. ${ }^{7}$

Phase analysis using gated SPECT or PET imaging has been used in a wide spectrum of cardiac disorders characterized by left ventricular dysfunction and heart failure. This can identify heart failure patients who might benefit from cardiac resynchronization therapy. ${ }^{8,9}$ This can also help optimize left ventricular lead position during resynchronization therapy ${ }^{10}$ and assess response to cardiac resynchronization therapy. ${ }^{11}$ Studies in patients with ischemic cardiomyopathy ${ }^{12}$ and non-ischemic cardiomyopathy ${ }^{13}$ show that left ventricular dyssynchrony is associated with a higher risk of mortality and portends a poor prognosis. Left ventricular dyssynchrony even in the absence of perfusion or function abnormalities has shown to be predict adverse cardiovascular outcomes in women ${ }^{14}$ and in patients with end stage renal disease. ${ }^{15}$

Phase analysis using gated SPECT is based on the concept of partial volume effect. ${ }^{7}$ The maximum counts from a region of the left ventricle are directly proportional to the myocardial thickness in that region. With a change in myocardial thickness during the cardiac cycle, there is a simultaneous change in counts. Measuring this change in counts during the cardiac cycle allows for an 
estimation of the degree and timing of thickening of different regions of the myocardium. ${ }^{7}$ Phase analysis involves using temporal 3D short axis frames of the left ventricular myocardium to assess the counts. ${ }^{7}$ Fourier transform processing is then applied to generate a regional wall thickening curve and a phase distribution in relation to the onset of mechanical contraction. ${ }^{7}$ Phase distribution helps demonstrate the uniformity or heterogeneity of myocardial thickening. The main indices of phase analysis, namely phase standard deviation and phase band width, are derived from the phase distribution, and have been validated in clinical studies as measures of dyssynchrony. ${ }^{7}$

The study by Malik et al. ${ }^{16}$ included patients with a history of diabetes mellitus for longer than 5 years and no previously known conduction abnormalities who underwent gated SPECT MPI, and were found to have normal perfusion and normal left ventricular function. Patient with a longer duration of diabetes mellitus and evidence of microvascular disease (diabetic retinopathy, microalbuminuria and/or neuropathy) showed higher standard deviation and phase width on phase distribution analysis. These patients were followed for approximately 2 years, and were subdivided into two groups based on the presence or absence of major adverse cardiovascular events (MACE). Over the two-year follow-up period, 9.5\% (13/136) developed MACE. Both parameters of left ventricular dyssynchrony were abnormal in patients with MACE when compared with those who did not develop MACE (phase standard deviation $16.0 \pm 9.4^{\circ}$ vs. $10.3 \pm 6.4^{\circ} ; \mathrm{p}=0.004$; phase band width $52.7 \pm 36.4^{\circ}$ vs $33.1 \pm 16.2^{\circ} ; \mathrm{p}=0.001$ ) On univariate analysis dyssynchrony was observed in patients with long-standing diabetes and evidence of microvascular disease but on multivariate analysis only duration of diabetes and microvascular disease were associated with dyssynchrony. This indicates dyssynchrony is perhaps a manifestation of long-standing diabetes and microvascular dysfunction in this population. This is an interesting study that describes the use of these novel parameters to identify diabetic patients at risk for MACE. The study does have limitations due to its small sample size.

Patients with diabetes mellitus who have normal perfusion and left ventricular function have an annual risk of $1-2 \%$ for cardiac death or non-fatal myocardial infarction, compared to less than $1 \%$ in non-diabetics with normal perfusion and left ventricular function. ${ }^{17}$ The MACE risk in this study was $9.5 \%$, which is considerably higher than expected based on previous data and the reasons for this are not clearly explained by the authors.

The correlation between higher MACE risk and left ventricular dyssynchrony in the setting of normal perfusion, left ventricular function, and conduction in patients with diabetes mellitus is probably reflective of endothelial dysfunction or microvascular disease in the coronary vascular bed. This opens up a discussion and platform for further research studies to investigate this finding further. If the results of this study can be corroborated at a larger scale, dyssynchrony analysis using gated SPECT might become a useful tool to study endothelial dysfunction and microvascular disease in larger patient populations.

Perhaps, we should consider incorporating dyssynchrony analysis in the routine interpretation of myocardial perfusion imaging studies along with myocardial perfusion, wall motion, and ejection fraction. However, this would require a standardization of the dyssynchrony parameters and a seamless incorporation of analysis algorithm in the programs used for interpretation of myocardial perfusion imaging studies in routine clinical practice.

\section{Disclosure}

Yogita Rochlani, Mohammed Hasan Khan, Perry Gerard, and Diwakar Jain have no relevant disclosures.

\section{References}

1. Garcia EV, Faber TL, Cooke CD, Folks RD, Chen J, Santana C. The increasing role of quantification in clinical nuclear cardiology: The Emory approach. J Nucl Cardiol 2007;14:420-32.

2. Germano G, Kavanagh PB, Slomka PJ, Van Kriekinge SD, Pollard G, Berman DS. Quantitation in gated perfusion SPECT imaging: The Cedars-Sinai approach. J Nucl Cardiol 2007;14:433-54.

3. Ficaro EP, Lee BC, Kritzman JN, Corbett JR. Corridor DM: The Michigan method for quantitative nuclear cardiology. J Nucl Cardiol 2007;14:455-65.

4. Watson DD, Smith WH. The role of quantitation in clinical nuclear cardiology: The University of Virginia approach. J Nucl Cardiol 2007;14:466-82.

5. Liu YH. Quantification of nuclear cardiac images: The Yale approach. J Nucl Cardiol 2007;14:483-91.

6. Chen J, Garcia E, Folks R, Cooke C, Faber T, Tauxe E, et al. Onset of left ventricular mechanical contraction as determined by phase analysis of ECG-gated myocardial perfusion SPECT imaging: Development of a diagnostic tool for assessment of cardiac mechanical dyssynchrony. J Nucl Cardiol 2005;12:68795.

7. Chen J, Garcia EV, Bax JJ, Iskandrian AE, Borges-Neto S, Soman P. SPECT myocardial perfusion imaging for the assessment of left ventricular mechanical dyssynchrony. J Nucl Cardiol 2011;18:685-94.

8. Boogers MM, Chen J, Bax JJ. Myocardial perfusion single photon emission computed tomography for the assessment of mechanical dyssynchrony. Curr Opin Cardiol 2008;23:431-9.

9. Boogers MM, Van Kriekinge SD, Henneman MM, Ypenburg C, Van Bommel RJ, Boersma E, et al. Quantitative Gated SPECTDerived Phase Analysis on Gated Myocardial Perfusion SPECT Detects Left Ventricular Dyssynchrony and Predicts Response to Cardiac Resynchronization Therapy. J Nucl Med 2009;50:718-25. 
10. Boogers MJ, Chen J, van Bommel RJ, Borleffs CJW, DibbetsSchneider P, van der Hiel B, et al. Optimal left ventricular lead position assessed with phase analysis on gated myocardial perfusion SPECT. Eur J Nucl Med Mol Imaging 2011;38:230-8.

11. Henneman MM, Chen J, Dibbets-Schneider P, Stokkel MP, Bleeker GB, Ypenburg C, et al. Can LV dyssynchrony as assessed with phase analysis on gated myocardial perfusion SPECT predict response to CRT? J Nucl Med 2007;48:1104-11.

12. AlJaroudi W, Alraies MC, Menon V, Brunken RC, Cerqueira MD, Jaber WA. Predictors and incremental prognostic value of left ventricular mechanical dyssynchrony response during stress-gated positron emission tomography in patients with ischemic cardiomyopathy. J Nucl Cardiol 2012;19:958-69.

13. Goldberg AS, Alraies MC, Cerqueira MD, Jaber WA, AlJaroudi WA. Prognostic value of left ventricular mechanical dyssynchrony by phase analysis in patients with non-ischemic cardiomyopathy with ejection fraction $35-50 \%$ and QRS $<150 \mathrm{~ms}$. J Nucl Cardiol 2014;21:57-66.
14. Sharma RK, Volpe G, Rosen BD, Ambale-Venkatesh B, Donekal S, Fernandes V, et al. Prognostic implications of left ventricular dyssynchrony for major adverse cardiovascular events in asymptomatic women and men: The multi-ethnic study of atherosclerosis. J Am Heart Assoc 2014;3:e000975.

15. Aggarwal H, AlJaroudi WA, Mehta S, Mannon R, Heo J, Iskandrian AE, et al. The prognostic value of left ventricular mechanical dyssynchrony using gated myocardial perfusion imaging in patients with end-stage renal disease. J Nucl Cardiol 2014;21:739_ 46.

16. Malik D, Mittal B, Sood A, Parmar M, Kaur K, Bahl A. Prognostic value of left ventricular mechanical dyssycnchrony incides in long-standing type II diabetes mellitus with normal perfusion and left ventricular systolic function on SPECT-MPI. J Nucl Cardiol Current Issue.

17. Bourque JM, Beller GA. Stress myocardial perfusion imaging for assessing prognosis: An update. JACC Cardiovasc Imaging 2011;4:1305-19. 